Viktor Meile, Maksym Tymkovych, Tobias Rusiecki, Yana Nosova, Florian Pape, Gerhard Poll, Birgit Glasmacher, Oleg Avrunin and Oleksandr Gryshkov*

\title{
Validation of a solvent-based process for the smoothing of additively manufactured 3D models of nasal cavities
}

\begin{abstract}
In order to improve the reliability of diagnosis of nasal breathing disorders, aerodynamic properties have to be analyzed through experiments based on 3D models. The surface properties of the prepared respective 3D models using fused deposition modeling (FDM) should match those of native nasal cavities, thus representing their normal state and typical pathologies. In this work, we validated the smoothing of dual extruded 3D printed samples of PLA (polylactide) and PVA (polyvinyl alcohol) using the solvent TFE (trifluoroethanol). The smoothing was conducted in vapour and liquid phases of TFE. Before and after treatment of the samples in liquid and vapour phases of TFE, mass and surface roughness analysis were performed. The results of this work will help to produce and process a representative model of the human paranasal sinuses, which can be created using CT data from a patient.
\end{abstract}

Keywords: 3D printing; fused deposition modeling; poly(lactic acid) (PLA); smoothing; processing; surface roughness

https://doi.org/10.1515/cdbme-2021-2107

\footnotetext{
*Corresponding author: Oleksandr Gryshkov: Institute for Multiphase Processes, Leibniz University Hannover, 30823 Garbsen, Germany; Lower Saxony Centre for Biomedical Engineering, Implant Research and Development, 30625 Hannover, Germany, e-mail: gryshkov@imp.uni-hannover.de Viktor Meile, Tobias Rusiecki: Institute for Multiphase Processes, Leibniz University Hannover, Garbsen, Germany Maksym Tymkovych, Yana Nosova, Oleg Avrunin: Department of Biomedical Engineering, Kharkiv National University of Radio Electronics, Kharkiv, Ukraine

Florian Pape, Gerhard Poll: Institute of Machine Design and Tribology, Leibniz University Hannover, Garbsen, Germany Birgit Glasmacher: Institute for Multiphase Processes, Leibniz University Hannover, Garbsen, Germany; Lower

Saxony Centre for Biomedical Engineering, Implant Research and Development, Hannover, Germany
}

\section{Introduction}

Chronic respiratory diseases are among the top four noncommunicable diseases (cardiovascular diseases, cancer, chronic respiratory diseases and diabetes). In 2012, 4.0 million people worldwide, or $10.7 \%$ of all noncommunicable diseases, died from chronic respiratory diseases, which include asthma and chronic obstructive pulmonary disease (COPD) [1].

Especially due to the pandemic that occurred in 2019 and the associated disease (COVID-19), the anamnesis and treatment of respiratory diseases takes on a new importance. Sinus diseases are becoming more common in today's world, and history recording and treatments are associated with caution and effort. Treatments of respiratory problems originating in the paranasal sinuses are usually pharmacotherapy options that relieve symptoms and, in some cases, even surgery (e.g., direction of the nasal septum) [2].

A more detailed history would increase the chances of success and alleviation of the patient's symptoms. In order to create a helpful simulation program, the aerodynamic characteristics of the airway and characteristics of the respiratory process must first be determined experimentally. In addition, airflow in the upper respiratory tract can be represented by laminar, turbulent streams and combination of both. Among other factors the surface roughness determines the type of stream, which was recently highlighted by Tabe et al. $[3,4]$.

Thus, the aim of this work was to investigate adequate post-processing methods for the smoothing of the surface of 3D printed samples using TFE as a solvent in its vapour and liquid phases. For this we developed experimental set ups, conducted a mass change study as well as analysis of the surface of the samples before and after smoothing. 


\section{Material and methods}

\subsection{Sample production and pre- processing}

An FDM printer with two extruders (3D printer, Ultimaker 3+ Extended) was utilized to obtain model PLA-PVA samples with the dimensions of $10 \mathrm{~mm} \times 10 \mathrm{~mm} \times 10 \mathrm{~mm}$. PVA served as a support material for the cavities (Figure 1) [5]. The following printing parameters were set: printing thickness of $0.1 \mathrm{~mm}$, line width of $0.35 \mathrm{~mm}$, printing temperature of $210^{\circ} \mathrm{C}$ and printing speed of $60 \mathrm{~mm} / \mathrm{s}$.

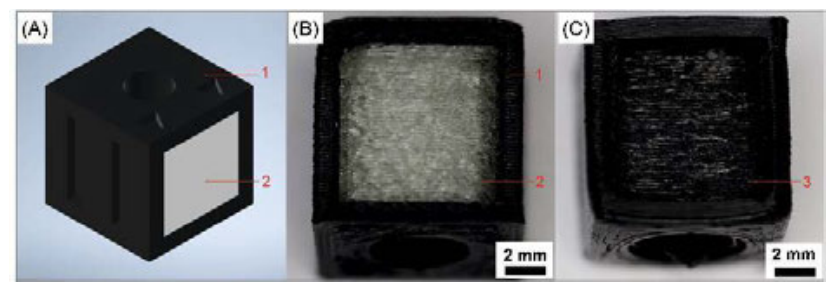

Figure 1: Illustration of the developed $C A D$ model $(A)$, the fabricated sample before (B) and after (C) PVA dissolution. (1) PLA main material, (2) PVA support material, (3) surface (8 $\mathrm{mm} \times 8 \mathrm{~mm}$ ) used for the surface roughness analysis.

After printing, the samples were placed in deionized water for $24 \mathrm{~h}$ and stirred constantly with a magnetic stirrer (RCT basic, IKA) at $1000 \mathrm{rpm}$ to dissolve the PVA component. Seven hours after the start of stirring and $7 \mathrm{~h}$ before the end the water was replaced. In order to remove the absorbed water, the samples were dried for $8 \mathrm{~h}$ at $37^{\circ} \mathrm{C}$ in the dryer (Binder CB150).

\subsection{Smoothing}

The solvent 2,2,2-trifluoroethanol; 99.8\% (TFE) was selected for smoothing experiments. Table 1 represents the smoothing durations as well as the number of samples measured.

Table 1: Smoothing durations and the number of samples measured for mass loss and analysis of the surface roughness.

\begin{tabular}{lll}
\hline Treatment duration, $\min$ & Liquid phase & Vapour phase \\
\hline 1 & $\mathrm{n}=5$ & - \\
2 & $\mathrm{n}=4$ & - \\
2.5 & $\mathrm{n}=3$ & $\mathrm{n}=3$ \\
3 & $\mathrm{n}=4$ & - \\
4 & $\mathrm{n}=5$ & - \\
\hline
\end{tabular}

\begin{tabular}{lll}
\hline Treatment duration, $\min$ & Liquid phase & Vapour phase \\
\hline 5 & $n=8$ & $n=5$ \\
10 & $n=4$ & $n=4$ \\
15 & $n=4$ & $n=4$ \\
20 & $n=4$ & $n=6$ \\
25 & $n=1$ & - \\
30 & $n=4$ & $n=3$ \\
$35 / 40 / 45 / 50 / 60$ & $n=1$ & - \\
60 & $n=1$ & $n=4$ \\
120 & $n=1$ & $n=2$ \\
$180 / 210$ & - & $n=1$ \\
240 & - & $n=2$ \\
$270 / 300$ & - & $n=1$ \\
\hline
\end{tabular}

In order to ensure repeatable smoothing procedures, smoothing chambers were developed (Figure 2). Samples were smoothed in liquid (Figure 2A) and vapour (Figure 2B) TFE in a $250 \mathrm{ml}$ glass cylinder filled with $100 \mathrm{ml}$ of TFE. To ensure a homogeneous mixing, the solvent was stirred with a magnetic stirrer at 200 (liquid) or 650 (vapour) rpm. The liquid smoothing was conducted at room temperature, whereas a temperature of $80^{\circ} \mathrm{C}$ was set during vapour smoothing experiments to accelerate solvent evaporation. A total number of 49 and 35 samples were smoothed using liquid and vapour methods, respectively.

Figure 2: Smoothing of the PCL samples in liquid (A) and vapour

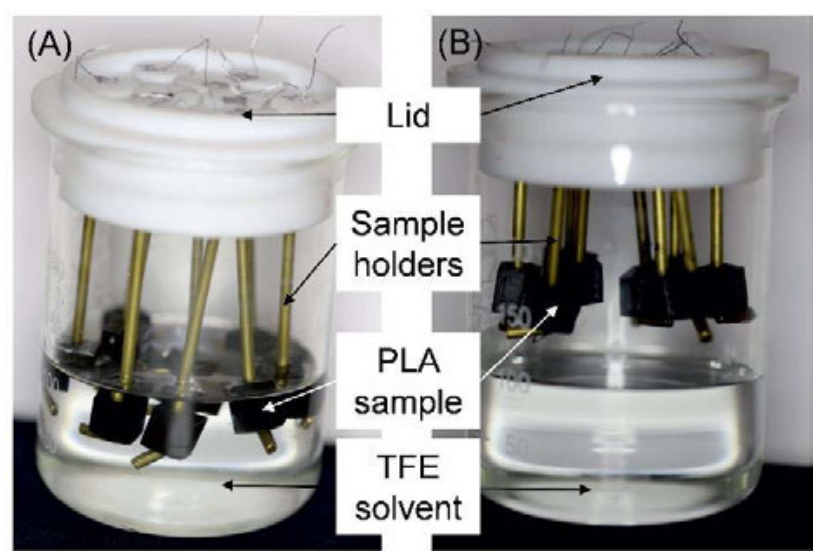

(B) phases of TFE.

\subsection{Analysis of the processed samples}

The samples' mass was measured before and after smoothing. Prior to the mass measurement, the samples were dried in the drying oven at $37{ }^{\circ} \mathrm{C}$ (Binder CB150) for $24 \mathrm{~h}$. The mass of each sample was measured before and after smoothing. The 
relative change in mass of a sample was calculated as the ratio between the mass of the same sample after and before smoothing.

Surface analysis was performed with a stereo microscope (100x, Discovery.V12 SteREO microscope, Carl Zeiss) and a confocal laser microscope (cLSM, 50x, VK-X200 series, Keyence). In case of cLSM, stitching of 32 images in an $8 \times 4$ grid was conducted. The images from the stereo microscope were analysed in the software "AxioVision" (Carl Zeiss, Rel. 4.7) using topography module, while the data from the cLSM were processed in the program "VK Analysis Module" (Keyence, version 3.8.0.0). In each case, the arithmetic mean value of the profile coordinates $R_{a}$ (surface roughness) was measured for three horizontal lines over a full width of a stitched image utilizing DIN EN ISO 4287:2010. For each group means and standard deviations were calculated while previously confirming a normal distribution using a ShapiroWilk normality test. The roughness of the PLA samples was analysed before and after smoothing.

\section{Results and discussion}

\subsection{Mass analysis}

The initial mean mass of the PLA samples before smoothing was $681 \pm 16 \mathrm{mg}(\mathrm{n}=84)$. Figure 3 represents the quantitative analysis of the sample mass change at different time points during smoothing in the vapour (Figure $3 \mathrm{~A}$, red open circles) and the liquid (Figure 3A, black circles) phases of TFE over a smoothing duration of $300 \mathrm{~min}$. The values were normalised on the mass of the untreated sample (dashed line on Figure 3). An insert in Figure 3 shows the change in mass for $30 \mathrm{~min}$ of smoothing. The results show a certain increase in the mass of the smoothed samples in the beginning of smoothing as compared to the untreated samples. This could result from a possible swelling of the printed PLA fibers and/or solvent uptake [6]. Additional experiments are needed to analyse the solvent content in the PLA sample upon smoothing.

In general, there is a clear trend that the vapour smoothing method does not noticeably affect the sample mass over a longer smoothing duration. Some decrease in mass can be observed after $240 \mathrm{~min}$ of smoothing in the vapour phase. In contrast to vapour smoothing, the liquid smoothing method resulted in a drastic decrease in mass of the sample after as few as $15 \mathrm{~min}$ of smoothing. As can be seen, in the range between 15 and 20 min of treatment, a 5\% decrease in mass is observed for liquid smoothing. The sample was completely dissolved after $180 \mathrm{~min}$ (Figure 3B). It should be noted that solution stirring of the TFE solvent in case of vapour smoothing could have contributed to a faster decrease in mass of the samples, as compared to gas smoothing.

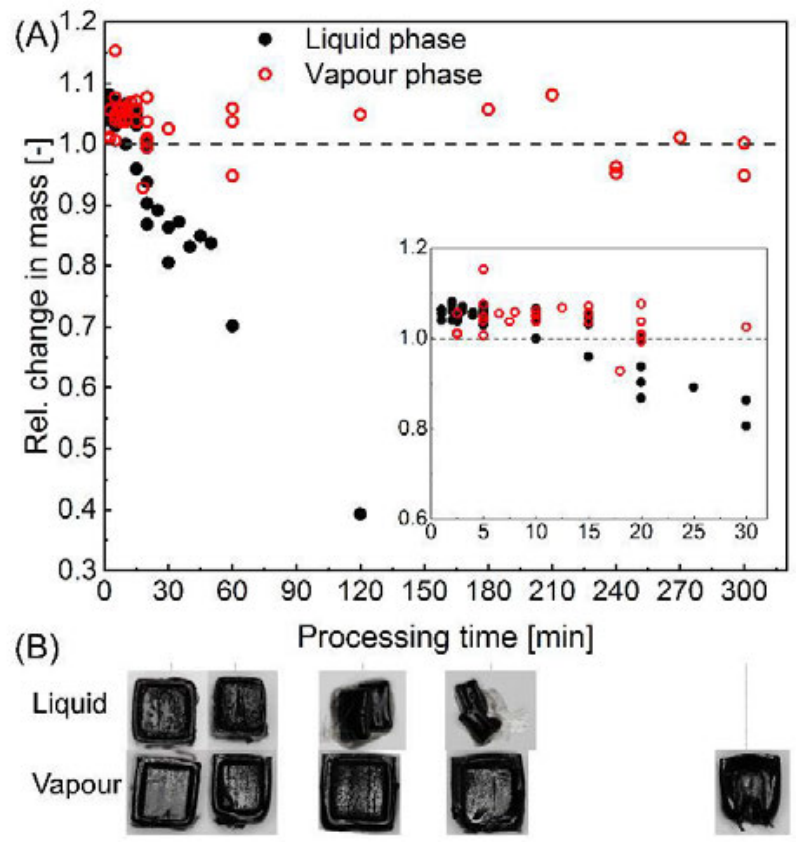

Figure 3: Relative change in mass of the PLA samples after smoothing in vapour (red open circles) and liquid (black circles) phases of TFE over a smoothing period of $300 \mathrm{~min}(\mathrm{~A})$. Insert in (A) shows a detailed view of the mass change over a smoothing period of $30 \mathrm{~min}$. The values were normalized on the mass of untreated sample (dashed lines). (B) Representative photographs of the smoothed samples.

\subsection{Surface analysis}

The arithmetic mean value of the profile coordinates $R_{a}$ indicates the arithmetic mean value of all deviations of the roughness profile from the mean line along the reference section. According to DIN EN ISO 4287:2010, the lower $R_{a}$, the smoother the surface. The analysis of the stereo microscope data revealed no clear correlation of the Ra values with respect to smoothing time as well as very broad Ra value distribution (data not shown). Thus, for more clarity only the cLSM results are shown.

Figure 4 shows the representative surface topography of the PLA sample before smoothing, analysed utilizing LSM. The colour scale bar represents a z-coordinate in $\mu \mathrm{m}$ of the measured profile. The mean roughness value of the surface of untreated samples was calculated to be $29 \pm 5 \mu \mathrm{m}$. Such variation in the roughness of the untreated samples can be attributed to the application of the PLA polymer from different batches for $3 \mathrm{D}$ printing of the models as well as possible changes in the temperature of the printing nozzles. 


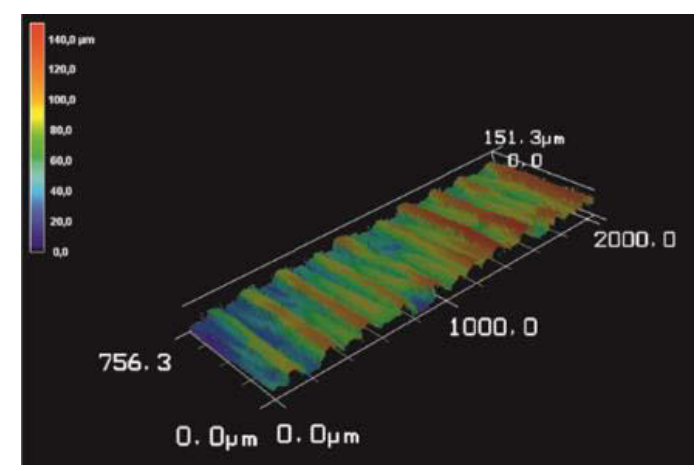

Figure 4: Representative surface topography of the PLA sample before smoothing using LSM.

After smoothing, the surface roughness decreased noticeably (Figure 5). In case of liquid smoothing, the surface roughness was calculated to be $7 \pm 3,9 \pm 6,16 \pm 9$ and $16 \pm 3 \mu \mathrm{m}$ after 2.5, 5.0, 10.0 and 15.0 min of smoothing. The surface roughness of PLA samples was $7 \pm 4,16 \pm 2,6 \pm 1$ and $14 \pm 2 \mu \mathrm{m}$ after 2.5, 5.0, 10.0 and $15.0 \mathrm{~min}$ of vapour smoothing.
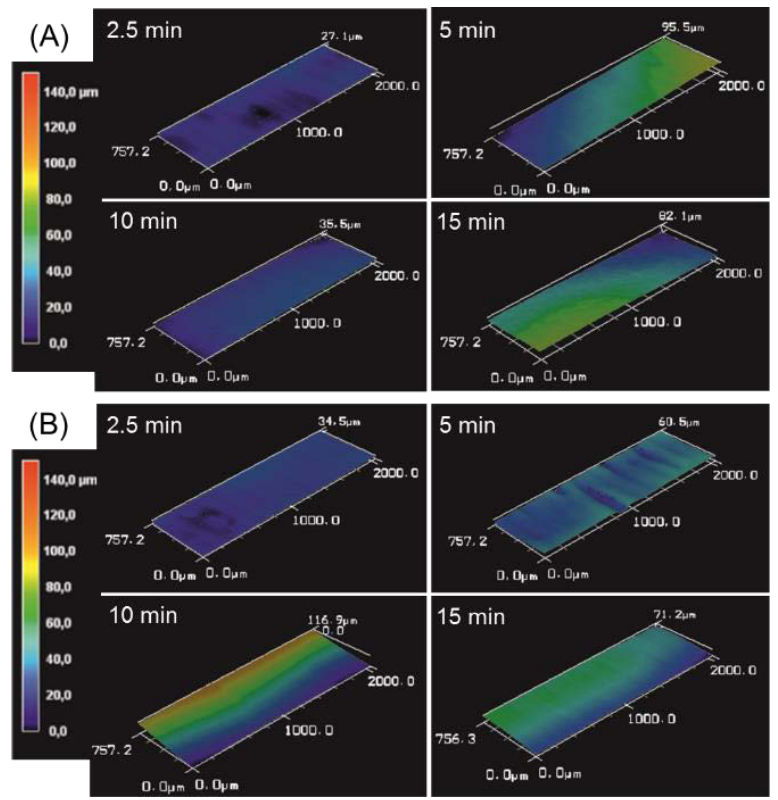

Figure 5: Surface topography of the PLA sample surface using LSM after smoothing in the vapour $(A)$ and liquid $(B)$ phases of TFE after 2.5, 5.0, 10.0 and 15.0 min of smoothing.

The results on measuring the $\mathrm{R}_{\mathrm{a}}$ did not follow a clear trend that would indicate that the surface roughness decreases (surface gets smoother) with increasing the smoothing time. In general, the results showed that the roughness values are reduced after a short time for both smoothing methods.

The qualitative analysis of the surface before and after smoothing was performed using vapour and liquid smoothing methods. Figure 5 shows the surface topography profiles of the sample surface after smoothing in vapour (Figure 5A) and liquid (Figure 5B) phases for the duration of 2.5, 5.0, 10.0 and $15.0 \mathrm{~min}$. As can be seen, the vapour smoothing seems to be more homogeneous, as compared to liquid smoothing.

\section{Conclusion and outlook}

The aim of this work was to validate the liquid and vapour methods for smoothing of the PLA 3D printed samples and to analyse the effect of smoothing and its duration on the mass change as well as surface properties. The results indicate that vapour smoothing is the best option to process the surfaces of a 3D printed polymeric materials to decrease the surface roughness while not changing the mass of the sample drastically. For smoothing in the liquid phase of TFE, treatment times of up to $15 \mathrm{~min}$ can also be recommended, where only a mass decrease of $5 \%$ was observed.

The results of this study show insights into how the surface treatment affects the surface characteristics of the 3D printed material. A more sophisticated experimental set up should be developed and validated for the smoothing of $3 \mathrm{D}$ models of the upper respiratory tracts should be developed, which can be reconstructed based on CT data of the patients.

\section{Author Statement}

Research funding: Joint German-Ukrainian project funded by BMBF (01DK20017) and the Ministry of Education and Science of Ukraine (100445538). Acknowledgements: SvenAlexander Barker for proof-reading of the manuscript. Conflict of interest: Authors declare no conflict of interest.

\section{References}

[1] World Health Organization: Global status report on noncommunicable diseases, 2014.

[2] Meltzer EO, Caballero F, Fromer LM, Krouse JH, Scadding G. Treatment of congestion in upper respiratory diseases. Int J Gen Med 2010;3:69-91.

[3] Tabe R, Rafee R, Valipour MS, Ahmadi G. Investigation of airflow at different activity conditions in a realistic model of human upper respiratory tract. Comput Methods Biomech Biomed Eng 2021:24(2):173-187.

[4] Prescher A. Klinische Anatomie der Nasennebenhöhlen. HNO 2009:57;1039-1052.

[5] Chia HN, Wu BM. Recent advances in 3D printing of biomaterials. J Biol Eng 2015:9;4, doi: 10.1186/s13036-0150001-4.

[6] Dong H, Esser-Kahn AP, Thakre PR, Patrick JF, Sottos NR, White SR, et al. Chemical treatment of poly(lactic acid) fibers to enhance the rate of thermal depolymerization. ACS Appl. Mater. Interfaces 2012:4(2):503-509. 